
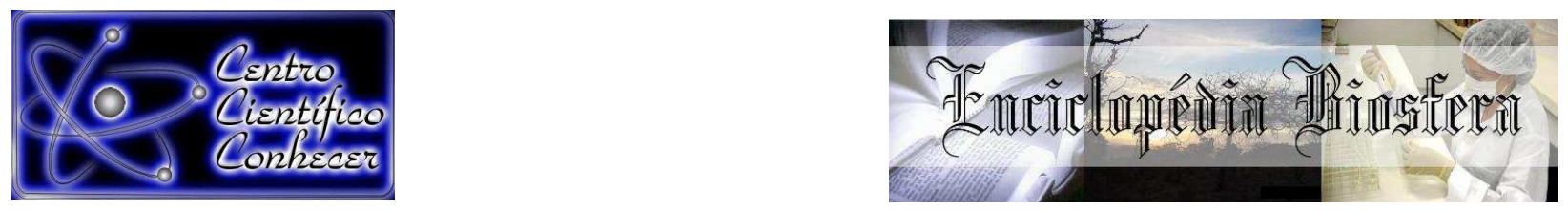

\title{
SAIS LIBERADORES DE ÓXIDO NÍTRICO NA EMERGÊNCIA E CRESCIMENTO DE SERINGUEIRA
}

Abimael Gomes da Silva ${ }^{1}$; Flávio Ferreira da Silva Binotti ${ }^{2}$; Edilson Costa ${ }^{3}$; Talita Cristina Campos Pereira ; Adriana Hernandes Pinto ${ }^{5}$

1 - Licenciado em Ciências Biológicas, mestrando na Universidade Estadual de Mato Grosso do Sul, UEMS, Unidade Universitária de Cassilândia

(maeldruida@hotmail.com);

2 - Agrônomo, Professor Adjunto UEMS, Unidade Universitária de Cassilândia;

3 - Engenheiro Agrícola, Professor Adjunto UEMS, Unidade Universitária de Cassilândia;

4 - Graduanda em Agronomia na Universidade Estadual de Mato Grosso do Sul, UEMS, Unidade Universitária de Cassilândia;

5 - Agrônoma, mestranda na Universidade Estadual de Mato Grosso do Sul, UEMS, Unidade Universitária de Cassilândia.

Recebido em: 08/04/2017 - Aprovado em: 10/06/2017 - Publicado em: 20/06/2017 DOI: 10.18677/EnciBio 2017A94

O fornecimento de sais inorgânicos liberadores de óxido nítrico podem colaborar para a emergência de algumas espécies resultando em um crescimento inicial diferenciado. Objetivou-se, com esse ensaio, avaliar a influência do fornecimento de sais inorgânicos liberadores de óxido nítrico via tratamento de semente por préembebição ou pela disponibilidade na primeira rega do substrato de emergência para 0 desenvolvimento inicial de seringueira. Utilizou-se 0 delineamento inteiramente casualizado, com seis tratamentos contendo quatro repetições cada, constituído por diferentes modos de fornecimento de sais inorgânicos (préembebição com água deionizada - controle 1, pré-embebição com nitrato de cálcio a $0,2 \%$, pré-embebição com nitrato de potássio a $0,2 \%$, fornecimento no substrato da emergência - água deionizada - controle 2, fornecimento no substrato da emergência com nitrato de cálcio a $0,2 \%$ e fornecimento no substrato da emergência com nitrato de potássio a $0,2 \%$ ). A pré-embebição consistiu da hidratação das sementes, contidas em recipientes plásticos por 24 horas em temperatura constante de $25^{\circ} \mathrm{C}$. O fornecimento no substrato da emergência, o mesmo foi hidratado em $100 \%$ da sua capacidade de campo em uma única aplicação das diferentes soluções. O fornecimento de sais de nitratos liberados de óxido nítrico não 
influenciaram a emergência de plântulas e fitomassas secas do vegetal, todavia a distribuição de fitomassa no vegetal foi influenciada, os tratamentos com nitrato de potássio no substrato e nitrato de cálcio via semente propiciaram maiores valores de comprimento do sistema radicular, diâmetro do caule, índice de qualidade de Dickson e menor RAD.

PALAVRAS-CHAVE: Hevea brasiliensis, nitrato de potássio, pré-embebição

\title{
NITRIC OXIDE RELEASES IN THE EMERGENCY OF RUBBER TREE
}

\begin{abstract}
The delivery of nitric oxide releasing inorganic salts may contribute to an emergence of species resulting in differentiated initial growth. The objective of this study was to evaluate the influence of the supply of nitric oxide releasing inorganic salts via seed treatment by pre-soaking or by the availability in the first irrigation of the emergency substrate for initial development of rubber tree. A completely randomized design was used, with 6 treatments containing 4 replicates each, consisting of different modes of inorganic salt supply (pre-soaking with deionized water - control 1, pre-soaking with $0.2 \%$ calcium nitrate, $0.2 \%$ potassium nitrate, supply in the emergency substrate deionized water - control 2 , supply in the emergency substrate with $0.2 \%$ calcium nitrate and supply in the emergency substrate with potassium nitrate at $0.2 \%)$. The pre-soaking consisted of hydration of the seeds, contained in plastic containers for 24 hours at a constant temperature of $25^{\circ} \mathrm{C}$. The supply in the emergency substrate was hydrated in $100 \%$ of its field capacity in a single application of the different solutions. The supply of nitrate salts released from nitric oxide did not influence the emergence of dry seedlings and plant phytomasses, however the distribution of phytomass in the plant was influenced, treatments with potassium nitrate in the substrate and calcium nitrate via seed provided higher values of Length of root system, stem diameter, Dickson quality index and lower RAD.
\end{abstract}

KEYWORDS: Hevea brasiliensis, potassium nitrate, pre-soaking

\section{INTRODUÇAO}

O petróleo, o minério e os produtos agrícolas, como a borracha natural são matérias primas que possuem grande importância no Brasil (FONTES, 2014). A borracha natural resulta do látex extraído de seringueiras e é convertida em diversos produtos, inclusive artigos da área da saúde: artigos médicos, luvas cirúrgicas, preservativos, pneus e calçados. Em alguns produtos é uma matéria substituível pela borracha sintética, no entanto, na maioria destes não pode ser substituída devido as características únicas (RIPPEL \& BRAGANÇA, 2009).

A seringueira é uma espécie com origem na Floresta Amazônica, do gênero Hevea e pertencente à família Euphorbiaceae. A Hevea brasiliensis [(Willd. ex. Adr. de Juss.) Muell Arg.] possui grande importância por ser a principal fonte de borracha natural explorada no mundo (LIU et al., 2015). 
A heveicultura é uma atividade com considerável retorno financeiro (NOGUEIRA et al., 2015) que tem ganhado espaço nas áreas de livre da doença do mal das folhas, como o estado de São Paulo. Como resultado, na cadeia produtiva há o aumento da procura por mudas de elevada qualidade. De acordo com normas vigentes para esse estado, exige-se que as mudas comerciais sejam produzidas em bancadas suspensas com uso de substratos livres de nematóides (BRASIL, 2009).

Mudas vigorosas são obtidas com o manejo adequado e com o uso de sementes com elevada capacidade de expressar seu potencial fisiológico. Muitos trabalhos com tratamentos pré-germinativos em sementes florestais objetivam superar a dormência, todavia, os tratamentos podem servir para agregar substâncias como sais inorgânicos que alteram os processos da germinação com distintos objetivos (MARCOS FILHO, 2015).

O óxido nítrico, NO, atua de diversas formas e auxilia muitos processos metabólicos no vegetal e pode ser obtido com o uso de sais do tipo nitrato. O NO está envolvido no desenvolvimento e crescimento da planta, em respostas ao estresse abiótico e na resistência aos patógenos (SANZ et al., 2015). De acordo com BINOTTI et al.,(2014) o uso de nitrato de potássio promoveu o aumento de velocidade de germinação, assim como o uso de nitrato de cálcio (BATISTA et al., 2016) em sementes de braquiária.

Esse estudo objetivou avaliar a influencia do fornecimento de sais inorgânicos liberadores de óxido nítrico via tratamento de semente por pré-embebição ou pela disponibilidade pela primeira rega no substrato na emergência de plântulas e crescimento inicial.

\section{MATERIAL E MÉTODOS}

Foi utilizado o delineamento experimental inteiramente casualizado, com seis tratamentos com quatro repetições cada, constituído por diferentes modos de fornecimento de sais inorgânicos (pré-embebição com água deionizada - grupo controle 1 (T1), pré-embebição com nitrato de cálcio a 0,2\% (T2), pré-embebição com nitrato de potássio a $0,2 \%$ (T3), fornecimento no substrato da emergência água deionizada - grupo controle 2 (T4), fornecimento no substrato da emergência nitrato de cálcio a $0,2 \%$ (T5) e fornecimento no substrato da emergência nitrato de potássio a $0,2 \%(T 6))$.

Nos tratamentos por fornecimento pela pré-embebição (T1 a T3), as sementes foram hidratadas em recipientes plásticos no período de 24 horas em temperatura constante de $25^{\circ} \mathrm{C}$. O fornecimento no substrato da emergência (T4 a T6), ocorreu com hidratação em $100 \%$ da capacidade de campo em uma única aplicação das diferentes soluções.

A semeadura ocorreu sete dias após a coleta das sementes. As avalições de crescimento foram realizadas aos 30 dias após semeadura, sendo que para as avaliações foram considerados os seguintes parâmetros:

Índice de velocidade de emergência (IVE) - As avaliações foram realizadas mediante a contagem diária do número de plântulas emergidas até estabilização e 0 ENCICLOPÉDIA BIOSFERA, Centro Científico Conhecer - Goiânia, v. 14 n.25; p. 137 
cálculo do índice de velocidade foi efetuado, calculado segundo a fórmula proposta por MAGUIRE (1962).

Foram mesurados: o comprimento do sistema radicular e da parte aérea do vegetal - aos 30 dias foi mensurado o comprimento do sistema radicular e da parte aérea vegetal, com o uso de régua graduada em $\mathrm{cm}$. Para a coleta dos dados, considerou-se 10 plantas por repetição e para as análises estatísticas foram utilizados os valores médios obtidos; diâmetro do caule: foi mensurado considerando 10 plântulas por repetição com o uso de paquímetro digital na região do colo do vegetal.

Fitomassas secas - na obtenção das fitomassas seca (fitomassa da parte aérea, do sistema radicular e total), as plantas consideradas normais tiveram a parte aérea e radicular separadas. Cada parte foi seca em estufa com circulação forçada de ar, regulada à temperatura de $65^{\circ} \mathrm{C}$, até atingir a massa constante. Em seguida, foi realizada a mensuração da massa em balança analítica. $O$ valor da fitomassa seca total foi obtido pela somatória dos resultados de fitomassa seca da parte aérea com a fitomassa seca do sistema radicular. Os resultados foram expressos em $\mathrm{g}$ plântula ${ }^{-1}$.

A partir dos parâmetros morfológicos foi obtido, por cálculos, as seguintes relações biométricas: Relação da altura da planta e a fitomassa seca da parte aérea - RAM; Relação da altura da planta e o diâmetro do colo - RAD; Relação da fitomassa seca da parte aérea e a fitomassa seca do sistema radicular - RMS; Índice de qualidade de Dickson - IQD (DICKSON et al., 1960), onde $I Q D=[M S T /(R A D+R M S)]$.

Todos os dados foram avaliados através da análise de variância pelo teste $F$, havendo significância entre os tratamentos utilizados foi aplicado o teste de Scottknott, todos a $5 \%$ de probabilidade.

\section{RESULTADOS E DISCUSSÃO}

$O$ índice de velocidade de emergência e a altura das plantas não foram influenciados pelos tratamentos utilizados (Tabela 1). De acordo com BATISTA et al., (2015) em ensaio com semente de pimenteira condicionadas, o uso do nitrato de cálcio, assim como de nitrato de potássio resultaram em velocidade de emergência semelhantes entre si e maior que o grupo controle.

TABELA 1 - Índice de velocidade de emergência (IVE), altura da parte aérea (AP), comprimento do sistema radicular (CR) e diâmetro de colo (DC) de plantas de seringueira em função do modo de fornecimento de sais inorgânicos, Cassilândia, MS, 2016 


\begin{tabular}{lcccc}
\hline Tratamento & IVE & AP $(\mathrm{cm})$ & CR $(\mathrm{cm})$ & DC $(\mathrm{mm})$ \\
\hline $\mathrm{KNO}_{3}$ (substrato) & $1,31 \mathrm{a}$ & $20,23 \mathrm{a}$ & $15,49 \mathrm{a}$ & $3,08 \mathrm{a}$ \\
$\mathrm{H}_{2} \mathrm{O}$ (substrato) & $1,05 \mathrm{a}$ & $21,10 \mathrm{a}$ & $14,48 \mathrm{~b}$ & $3,02 \mathrm{a}$ \\
$\mathrm{Ca}\left(\mathrm{NO}_{3}\right)_{2}$ (substrato) & $1,12 \mathrm{a}$ & $21,14 \mathrm{a}$ & $15,63 \mathrm{a}$ & $1,63 \mathrm{c}$ \\
$\mathrm{Ca}\left(\mathrm{NO}_{3}\right)_{2}$ (semente) & $1,27 \mathrm{a}$ & $22,17 \mathrm{a}$ & $16,23 \mathrm{a}$ & $3,18 \mathrm{a}$ \\
$\mathrm{H}_{2} \mathrm{O}$ (semente) & $1,26 \mathrm{a}$ & $21,38 \mathrm{a}$ & $13,57 \mathrm{~b}$ & $3,23 \mathrm{a}$ \\
$\mathrm{KNO}_{3}$ (semente) & $1,30 \mathrm{a}$ & $19,96 \mathrm{a}$ & $11,92 \mathrm{c}$ & $2,64 \mathrm{~b}$ \\
\hline C.V.(\%) & 14,15 & 8,27 & 2,92 & 4,23 \\
\hline
\end{tabular}

Médias seguidas de mesma letra na coluna não diferem entre si pelo teste de Scott-knott a $5 \%$ de probabilidade.

O comprimento do sistema radicular apresentou o menor valor para o uso de nitrato de potássio administrado via tratamento de semente. Entre os tratamentos de semente, o uso de nitrato de cálcio promoveu raízes com maior comprimento. $\mathrm{O}$ tratamento com os sais fornecido no substrato resulta em raízes maiores do que as que tiveram a primeira regada sem nitrato. Dentre todos os tratamentos, o uso de nitrato de cálcio via substrato resultou no menor diâmetro de colo. Comparando os tratamentos de semente, o uso de nitrato de potássio resultou em menor diâmetro comparado ao nitrato de cálcio e ao grupo controle.

As fitomassas secas da parte aérea, do sistema radicular e total não apresentaram diferença estatística para os tratamentos utilizados de acordo com a Tabela 2. Esses resultados são semelhantes aos encontrados por NUNES et al. (2015) com uso de nitrato de potássio no tratamento de sementes de crambe. No entanto, segundo BATISTA et al., (2015) o uso dos nitratos no condicionamento de sementes de pimenteira resultou no incremento da fitomassa seca.

TABELA 2 - Fitomassa seca da parte aérea (FSA), fitomassa seca do sistema radicular (FSR), fitomassa seca total (FST) de plantas de seringueira em função do modo de fornecimento de sais inorgânicos, Cassilândia, MS, 2016

\begin{tabular}{lccc}
\hline \multicolumn{1}{c}{ Tratamento } & FSA $(\mathrm{g})$ & $\mathrm{FSR}(\mathrm{g})$ & $\mathrm{FST}(\mathrm{g})$ \\
\hline $\mathrm{KNO}_{3}$ (substrato) & $0,53 \mathrm{a}$ & $0,22 \mathrm{a}$ & $0,75 \mathrm{a}$ \\
$\mathrm{H}_{2} \mathrm{O}$ (substrato) & $0,47 \mathrm{a}$ & $0,22 \mathrm{a}$ & $0,70 \mathrm{a}$ \\
$\mathrm{Ca}\left(\mathrm{NO}_{3}\right)_{2}$ (substrato) & $0,50 \mathrm{a}$ & $0,24 \mathrm{a}$ & $0,73 \mathrm{a}$ \\
$\mathrm{Ca}\left(\mathrm{NO}_{3}\right)_{2}$ (semente) & $0,60 \mathrm{a}$ & $0,20 \mathrm{a}$ & $0,80 \mathrm{a}$ \\
$\mathrm{H}_{2} \mathrm{O}$ (semente) & $0,61 \mathrm{a}$ & $0,22 \mathrm{a}$ & $0,83 \mathrm{a}$ \\
$\mathrm{KNO}_{3}$ (semente) & $0,54 \mathrm{a}$ & $0,20 \mathrm{a}$ & $0,73 \mathrm{a}$ \\
\hline C.V.(\%) & 14,58 & 22,73 & 15,89 \\
\hline
\end{tabular}

Médias seguidas de mesma letra na coluna não diferem entre si pelo teste de Scott-knott a $5 \%$ de probabilidade.

Estão demonstradas na Tabela 3 a relação da fitomassa seca da parte aérea e fitomassa seca do sistema radicular (RMS), relação da altura da parte aérea e diâmetro do colo $(R A D)$, relação da altura da planta e a fitomassa seca da parte 
aérea (RAM) e índice de qualidade de Dickson (IQD). O nitrato de cálcio administrado via tratamento de semente apresentou valor superior de RMS, quando comparado com os tratamentos em que a primeira rega consistiu de solução nitrato de cálcio e controle - água.

TABELA 3 - Relação da fitomassa seca da parte aérea e fitomassa seca do sistema radicular (RMS), relação da altura da parte aérea e diâmetro do colo (RAD) relação da altura da planta e a fitomassa seca da parte aérea (RAM) e índice de qualidade de Dickson (IQD) de plantas de seringueira em função do modo de fornecimento de sais inorgânicos, Cassilândia, MS, 2016

\begin{tabular}{lcccc}
\hline Tratamento & RMS $\left(\mathrm{g} . \mathrm{g}^{-1}\right)$ & $\mathrm{RAD}\left(\mathrm{cm} \cdot \mathrm{mm}^{-1}\right)$ & $\mathrm{RAM}\left(\mathrm{cm} \cdot \mathrm{g}^{-1}\right)$ & IQD \\
\hline $\mathrm{KNO}_{3}$ (substrato) & $2,42 \mathrm{ab}$ & $6,57 \mathrm{~b}$ & $38,44 \mathrm{a}$ & $0,08 \mathrm{a}$ \\
$\mathrm{H}_{2} \mathrm{O}$ (substrato) & $2,11 \mathrm{~b}$ & $6,99 \mathrm{~b}$ & $45,93 \mathrm{a}$ & $0,08 \mathrm{a}$ \\
$\mathrm{Ca}\left(\mathrm{NO}_{3}\right)_{2}$ (substrato) & $2,14 \mathrm{~b}$ & $13,02 \mathrm{a}$ & $43,59 \mathrm{a}$ & $0,05 \mathrm{~b}$ \\
$\mathrm{Ca}\left(\mathrm{NO}_{3}\right)_{2}$ (semente) & $3,24 \mathrm{a}$ & $6,97 \mathrm{~b}$ & $38,16 \mathrm{a}$ & $0,08 \mathrm{a}$ \\
$\mathrm{H}_{2} \mathrm{O}$ (semente) & $2,75 \mathrm{ab}$ & $6,62 \mathrm{~b}$ & $35,34 \mathrm{a}$ & $0,09 \mathrm{a}$ \\
$\mathrm{KNO}_{3}$ (semente) & $2,83 \mathrm{ab}$ & $7,58 \mathrm{~b}$ & $37,22 \mathrm{a}$ & $0,07 \mathrm{ab}$ \\
\hline C.V.(\%) & 17.04 & 11.00 & 15.90 & 20.61 \\
\hline
\end{tabular}

Médias seguidas de mesma letra na coluna não diferem entre si pelo teste de Scott-knott a $5 \%$ de probabilidade.

Os valores de RAD foram maiores no tratamento via primeira rega com nitrato de cálcio quando comparado aos demais tratamentos utilizados. Os valores de RAM não apresentaram diferença estatística entre os tratamentos. $O$ uso dos nitratos via semente não diferiram estatisticamente entre si para o índice de qualidade de Dickson. Para essa mesma variável, a administração de nitrato de cálcio via primeira rega resultou no menor valor, todavia não diferindo com o fornecimento de nitrato de potássio via semente.

Os índices biométricos demonstram a qualidade do vegetal, levando em consideração aspectos arquitetônicos como todo e são mais precisos do que dados morfológicos isolados. De acordo com PEREIRA et al. (2016) a relação da altura da parte aérea pelo diâmetro do colo é um índice que permite averiguar crescimentos irregulares, mudas estioladas apresentam resultados elevados para essa relação. $O$ índice de qualidade de Dickson varia de acordo com a idade das mudas, sendo recomendados os valores que sejam maiores do que 0,2 . Para esse trabalho, não foi possível chegar a esse índice tendo em vista que as plantas estavam com 30 dias no período de avaliação.

\section{CONCLUSÕES}

O fornecimento de sais de nitratos, liberadores de óxido nítrico, não influenciou a emergência das plantas e fitomassas secas do vegetal, todavia a distribuição de fitomassa no vegetal foi influenciada. Os tratamentos com nitrato de 
potássio no substrato ou nitrato de cálcio via semente propiciaram maiores valores de comprimento do sistema radicular, diâmetro do caule, índice de qualidade de Dickson e menor relação da altura da parte aérea e diâmetro do colo.

\section{AGRADECIMENTOS}

Os autores agradecem à Fundação de Apoio ao Desenvolvimento do Ensino, Ciência e Tecnologia do Estado do Mato Grosso do Sul (Fundect) pela bolsa concedida ao primeiro e quarto autor, e pelo apoio financeiro (FUNDECT/CNPq/PRONEM - MS, Processo 59/300.116/2015 - № FUNDECT 080/2015).

\section{REFERENCIAS}

BATISTA, T. B.; BINOTTI, F. F. S.; CARDOSO, E.D.; BARDIVIESSO, E. M.; COSTA, E.; Aspectos fisiológicos e qualidade de mudas da pimenteira em resposta ao vigor e condicionamento das sementes. Bragantia, Campinas, v. 74, n. 4, p. 367-373, 2015. Disponível em: <http://dx.doi.org/10.1590/1678-4499.0133>. doi: 10.1590/16784499.0133

BATISTA, T. B.; CARDOSO, E.D.; BINOTTI, F. F. S.; COSTA, E.; SÁ, M. E. Priming and stress under high humidity and temperature on the physiological quality of Brachiaria brizantha cv. MG-5 seeds. Acta Scientiarum, Maringá, v. 38, n. 1, p. 123127, 2016. Disponível em: <http://dx.doi.org/10.4025/actasciagron.v38i1.26021>. doi: 10.4025/actasciagron.v38i1.26021

BINOTTI, F. F. S.; SUEDA JUNIOR, C. I.; CARDOSO, E. D.; HAGA, K. I. NOGUEIRA D. C. Tratamentos pré-germinativos em sementes de Brachiaria. Revista Brasileira de Ciências Agrárias, Recife, v.9, n.4, p.614-618, 2014. Disponível em: <http://www.agraria.pro.br/sistema/index.php?journal=agraria\&page=article\&op=view \&path\%5B\%5D=agraria_v9i4a2781>. doi: 10.5039/agraria.v9i4a2781

BRASIL. Ministério da Agricultura, Pecuária e Abastecimento (MAPA). Instrução Normativa no 29, de 05 de agosto de 2009. Aprova as normas para a produção de sementes e de mudas de seringueira (Hevea spp.). Diário Oficial da República Federativa do Brasil, Brasília-DF, 06/08/2009. Seção 1, p. 5. Disponível em: <http://www.ciflorestas.com.br/arquivos/doc_normas_seringueira_26735.pdf> ISSN 1677-7042

DICKSON, A.; LEAF, A. L.; HOSNER, J. F. Quality appraisal of white spruce and white pine seedling stock in nurseries. Forestry Chronicle, Ottawa, v. 36, p. 10-13, 1960. Disponível em: <http://pubs.cif-ifc.org/doi/pdf/10.5558/tfc36010-1>. doi: 10.5558/tfc36010-1 
FONTES, M. S. Recursos naturais nas Relações Internacionais: O precedente do Brasil na Segunda Guerra Mundial. Passagens: Revista Internacional de História Política e Cultura Jurídica Rio de Janeiro, v. 7, n. 1, 2015, p. 109-132. Disponível em: <http://www.revistapassagens.uff.br/index.php/Passagens/article/view/30/28>. doi: 10.5533/1984-2503-20157106

LIU, S.; LAN, J.; ZHOU, B.; QIN, Y.; ZHOU, Y.; XIAO, X.; YANG, J.; GOU, J.; QI, J.; HUANG, Y.; TANG, C. HbNIN2, a cytosolic alkaline/neutral-invertase, is responsible for sucrose catabolism in rubber-producing laticifers of Hevea brasiliensis (para rubber tree). New Phytologist, Lancaster, v. 206, n. 2, p. 709-725, 2015. Disponível em: <http://onlinelibrary.wiley.com/doi/10.1111/nph.13257/abstract>. doi: $10.1111 /$ nph. 13257

MARCOS FILHO, J. Fisiologia de sementes de plantas cultivadas. Piracicaba, SP: FEALQ, 2015. 655 p.

MAGUIRE, J. D. Speed of germination and in selection and evaluation for seedling emergence and vigor. Crop Science, v.2, n.2, p.176-177, 1962. Disponível em: <https://dl.sciencesocieties.org/publications/cs/abstracts/2/2/CS0020020176>.

NOGUEIRA, R. F.; CORDEIRO, S. A.; LEITE, A. M. P.; BINOTI, M. L. M. S.. Mercado de borracha natural e viabilidade econômica do cultivo da seringueira no Brasil. Nativa, v. 03, n. 02, p. 143-149, 2015. Disponível em: <http://dx.doi.org/10.14583/2318-7670.v03n02a12>. doi: 10.14583/23187670.v03n02a12

NUNES, A. S.; SOUZA, L. C. F.; SCALON, S. P. Q.; PAGNONCELLI, J. Nitrato de potássio e retirada do pericarpo na germinação e na avaliação do vigor de sementes de crambe. Semina: Ciências Agrárias, Londrina, v. 36, n. 3, p. 1775-1782, 2015. Disponível

em: <http://www.uel.br/revistas/uel/index.php/semagrarias/article/view/12627>. doi: 10.5433/1679-0359.2015v36n3Supl1p1775

PEREIRA, K. T. O.; OlIVEIRA, F. A.; CAVALCANTE, A. L. G.; DANTAS, R. P.; OLIVEIRA, M. K. T.; COSTA, J. P. B. M. Qualidade de mudas de moringa sob diferentes níveis de nutrientes aplicados via fertirrigação. Pesquisa Florestal Brasileira, Colombo, v. 36, n. 88, p. 497-504, 2016. Disponível em: < http://dx.doi.org/10.4336/2016.pfb.36.88.1038>. doi: 10.4336/2016.pfb.36.88.1038

RIPPEL, M. M.; BRAGANÇA, F. C. Borracha natural e nanocompósitos com argila. Química Nova, São Paulo, SP, v. 32, n. 3, p. 818-826, mar. 2009. Disponível em: <http://quimicanova.sbq.org.br/imagebank/pdf/Vol32No3_818_23-QN09043.pdf>.

SANZ, L.; ALBERTOS, P.; MATEOS, I.; SÁNCHEZ-VICENTE, I.; LECHÓN, T.; FERNÁNDEZ-MARCOS, M.; LORENZO, O. Nitric oxide (NO) and phytormones crosstalk during early plant development. Journal of Experimental Botany, v. 66, n. 10, p. 2857-2868, 2015. Disponível em: <https://doi.org/10.1093/jxb/erv213>. doi: 10.1093/jxb/erv213 